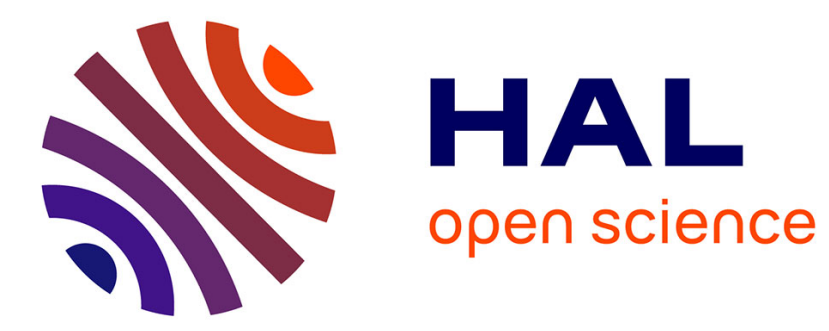

\title{
Foreground Detection using the Choquet Integral
}

Fida El Baf, Thierry Bouwmans, Bertrand Vachon

\section{To cite this version:}

Fida El Baf, Thierry Bouwmans, Bertrand Vachon. Foreground Detection using the Choquet Integral. 9th International Workshop on Image Analysis for Multimedia Interactive Services, May 2008, Klagenfurt, Austria. pp.187-190, 10.1109/WIAMIS.2008.9 . hal-00333062

\section{HAL Id: hal-00333062 \\ https://hal.science/hal-00333062}

Submitted on 22 Oct 2008

HAL is a multi-disciplinary open access archive for the deposit and dissemination of scientific research documents, whether they are published or not. The documents may come from teaching and research institutions in France or abroad, or from public or private research centers.
L'archive ouverte pluridisciplinaire HAL, est destinée au dépôt et à la diffusion de documents scientifiques de niveau recherche, publiés ou non, émanant des établissements d'enseignement et de recherche français ou étrangers, des laboratoires publics ou privés. 


\title{
Foreground Detection using the Choquet Integral
}

\author{
$\begin{array}{lll}\text { F. EL BAF T. BOUWMANS } \quad \text { B. VACHON } & \end{array}$ \\ University of La Rochelle - France \\ Laboratory of Mathematics, Images and Applications \\ E-mail: felbaf@univ-lr.fr
}

\begin{abstract}
Foreground Detection is a key step in background subtraction problem. This approach consists in the detection of moving objects from static cameras through a classification process of pixels as foreground or background. The presence of some critical situations i.e noise, illumination changes and structural background changes produces an uncertainty in the classification of image pixels which can generate false detections. In this context, we propose a fuzzy approach using the Choquet integral to avoid the uncertainty in the classification. The experiments on different video datasets have been realized by testing different color space and by fusing color and texture features. The proposed method is characterized through robustness against illumination changes, shadows and little background changes, and it is validated with the experimental results.
\end{abstract}

\section{Introduction}

Analysis and understanding of video sequences is an active research field in computer vision due to its applicability in diverse discipline. Important applications of moving object detection includes automatic video surveillance system [3], optical motion capture [1], multimedia application [4] and so on. Such kind of applications need to identify person, car or other moving objects in the scene. So, the basic operation needed is the separation of the moving objects called foreground from the static information called background. The process considered as unavoidable part for this kind of operation relies on background subtraction based techniques. In the literature, many background subtraction methods can be found to handle the illumination changes and other background changes met in video sequences. These different methods are classified following the model used:

- Basic Background Modeling: In this case, Background Representation is modeled using the average [7], the median [9] or the histogram analysis over time [18]. Once the model is computed, the foreground detection is made as follows:

$$
d\left(I_{t}(x, y, t)-B_{t}(x, y, t-1)\right)>T
$$

Otherwise, pixels are classified as background.

$T$ is a constant threshold, $I_{t}(x, y, t)$ and $B_{t}(x, y, t)$ are respectively the current and the background images.

- Statistical Background Modeling: Background Representation is modeled using a single Gaussian [16], a Mixture of Gaussians [12] or a Kernel Density Estimation [5]. Statistical variables are used in the foreground detection to classify the pixels as foreground or background.

- Background Estimation: Background Representation is estimated using a filter. For the foreground detection, any pixel of the current image that deviates significantly from its predicted value is declared foreground. This filter may be a Wiener filter [15], a Kalman filter [10] or a Tchebychev filter [2].

In these different approaches, the features commonly used to handle critical situations are color, edge, stereo, motion and texture. Often, these features are used separately and the most used is the color one. The combination of several measuring features can strengthen the pixels classification as background or foreground. In a general way, the Choquet and Sugeno integrals have been successfully applied widely in classification problems [14], in decision making [11] and also in data modelling [13] to aggregate different measures. In the context of foreground detection, these integrals seem to be good candidates for fusing different measures from different features. Each integral has its particularity. Recently, Zhang and Xu [17] have used color features obtained from Ohta color space and texture features to obtain similarity measures which are aggregated using the Sugeno integral. The assumption made by the authors reflects that the scale is ordinal. Then, the objects are detected by thesholding the results of the Sugeno integral. In 
this work, the scheme used is based on Xu's algorithm. But since the scale is continuum in the foreground detection, the Choquet integral seems to be more suitable than Sugeno integral. So, we propose to use the Choquet integral to aggregate color and texture features instead of the Sugeno integral. Then, the algorithm was improved by testing different color spaces which are more robust to shadows and illumination changes due to their geometrical characteristics. The paper is organized as follows: In section 2, we introduce the features and the similarity measures adopted. Section 3 defines the fuzzy integrals. The proposed method for the foreground detection is involved in section 4. Finally, the last section compares our method with the algorithm proposed by Zhang and $\mathrm{Xu}$ [17] using videos datasets from multimedia and video surveillance applications.

\section{Color and Textures Similarity Measures}

Foreground detection is based on a comparison between current and background images. In general, a simple subtraction is made between these two images to detect regions corresponding to foreground. Another way to establish this comparison consist in defining a similarity measure between pixels in current and background images. In this case, pixels corresponding to background should be similar in the two images while pixels corresponding to foreground should not be similar. The computing of the similarity measures can be done using the color features. For more robustness against illumination changes, we propose in the following subsections to compute similarity for texture feature too.

\subsection{Color Similarity Measure}

In the following, we describe the similarity measure in a general way, i.e the color features may be any color space with three components noted $I_{1}, I_{2}$ and $I_{3}$. Then, the color similarity measure $S_{k}^{C}(x, y)$ at the pixel $(x, y)$ is computed as in [17]:

$$
S_{k}^{C}(x, y)=\left\{\begin{array}{lll}
\frac{I_{k}^{C}(x, y)}{I_{k}^{B}(x, y)} & \text { if } & I_{k}^{C}(x, y)<I_{k}^{B}(x, y) \\
1 & \text { if } & I_{k}^{C}(x, y)=I_{k}^{B}(x, y) \\
\frac{I_{k}^{B}(x, y)}{I_{k}^{C}(x, y)} & \text { if } & I_{k}^{C}(x, y)>I_{k}^{B}(x, y)
\end{array}\right.
$$

where $k \in\{1,2,3\}$ is one of the three color features, $B$ and $C$ represent respectively the background and the current images at time $t$. $B$ can be obtained using any of the background modelling method. Note that $S_{k}^{C}(x, y)$ is between 0 and 1 . Futhermore, $S_{k}^{C}(x, y)$ is closed to one if $I_{k}^{C}(x, y)$ and $I_{k}^{B}(x, y)$ are very similar.

\subsection{Texture Similarity Measure}

The texture features used is the Local Binary Pattern (LBP) which is developed by Heikkila and Pietikinen [6]. The LBP is invariant to monotonic changes in grey scale, which makes it robust against illumination changes. The operator labels the pixels of an image block by thresholding the neighbourhood of each pixel with the center value and considering the result as a binary number:

$$
L B P(x, y)=\sum_{i=0}^{N-1} s\left(g_{i}-g\right) 2^{i}
$$

where $g$ corresponds to the grey value of the center pixel $(x, y)$ and $g_{i}$ to the grey values of the $N$ neighbourhood pixels. The function is defined as follows:

$$
s(x)=\left\{\begin{array}{lll}
1 & \text { if } & x \geq 0 \\
0 & \text { if } & x<0
\end{array}\right.
$$

The original LBP operator worked with the $3 \times 3$ neighbourhood of a pixel.

Then, the texture similarity measure $S^{T}(x, y)$ at the pixel $(x, y)$ is computed as follows:

$$
S^{T}(x, y)=\left\{\begin{array}{lll}
\frac{L^{C}(x, y)}{L^{B}(x, y)} & \text { if } & L^{C}(x, y)<L^{B}(x, y) \\
1 & \text { if } & L^{C}(x, y)=L^{B}(x, y) \\
\frac{L^{B}(x, y)}{L^{C}(x, y)} & \text { if } & L^{C}(x, y)>L^{B}(x, y)
\end{array}\right.
$$

where $L^{B}(x, y)$ and $L^{C}(x, y)$ are respectively the texture LBP of pixel $(x, y)$ in the background and current images at time $t$. Note that $S^{T}(x, y)$ is between 0 and 1 . Furthermore, $S^{T}(x, y)$ is close to one if $L^{B}(x, y)$ and $L^{C}(x, y)$ are very similar.

\section{Fuzzy Integrals}

In this section, we summarize briefly necessary concepts around fuzzy integrals (Sugeno and Choquet) and the difference between them.

Let $\mu$ be a fuzzy measure on a finite set $X$ of criteria and $h: X \rightarrow[0,1]$ be a fuzzy subset of $X$.

Definition 1 The Sugeno integral of $h$ with respect to $\mu$ is defined by:

$$
S_{\mu}=\operatorname{Max}\left(\operatorname{Min}\left(h\left(x_{\sigma(i)}\right), \mu\left(A_{\sigma(i)}\right)\right)\right)
$$

where $\sigma$ is a permutation of the indices such that $h_{\sigma(1)} \leq \ldots \leq h_{\sigma(n)}$ and $A_{\sigma(i)}=\{\sigma(1), \ldots, \sigma(n)\}$ 
Definition 2 The Choquet integral of $h$ with respect to $\mu$ is defined by:

$$
C_{\mu}=\sum_{i=0}^{n} h\left(x_{\sigma(i)}\right)\left(\mu\left(A_{\sigma(i)}\right)-\mu\left(A_{\sigma(i+1)}\right)\right)
$$

with the same notations as above.

One of the properties of these integrals is that Choquet integral is adapted for cardinal aggregation while Sugeno integral is more suitable for ordinal aggregation (more details can be found in ([14],[11],[13])).

\section{Aggregation of color and texture similarity measures by Choquet Integral}

The numbers have a real meaning with Choquet integral while only order makes sense in Sugeno integral. Since the scale is continuum in the foreground detection, the Choquet integral seems to be more suitable than Sugeno integral, that is why we opted for Choquet integral in this section to aggregate different features. So, for each pixel, color and texture similarity measures are computed as explained in section 2 from the background and the current frame. We define the set of criteria $X \in\left\{x_{1}, x_{2}, x_{3}\right\}$ with, $\left(x_{1}, x_{2}\right)=$ two components color features of the chosen color space (i.e. Ohta, HSV, YCrCb etc) and $x_{3}=$ texture feature obtained by the LBP. For each $x_{i}$, let $\mu\left(x_{i}\right)$ be the importance that takes the feature $x_{i}$ in the decision of the foreground detection process. The fuzzy functions $h\left(x_{i}\right)$ are defined in $[0,1]$ so that, $h\left(x_{1}\right)=S_{1}^{C}(x, y)$, $h\left(x_{2}\right)=S_{2}^{C}(x, y)$ and $h\left(x_{3}\right)=S^{T}(x, y)$. To compute the value of Choquet integral for each pixel, we need firstly to rearrange the features $x_{i}$ in the set $X$ with respect to the order: $h\left(x_{1}\right) \geq h\left(x_{2}\right) \geq h\left(x_{2}\right)$.

The pixel at position $(x, y)$ is considered as foreground if its Choquet integral value is less than a certain threshold $T h$ (pixels not similar):

$$
\text { if } C_{\mu}(x, y)<T h \text { then }(x, y) \text { is foreground. }
$$

\section{Experimental results}

We have applied our algorithm to different datasets: the first one is our Aqu@theque dataset used in a multimedia application [4], the second one is the VS-PETS $2003{ }^{1}$ used in video sport application and the PETS $2006^{2}$ dataset applicated in video surveillance. We provide results of one video of each datasets without post processing. The background image is obtained using the running average model.

\footnotetext{
${ }^{1}$ http://ftp.pets.rdg.ac.uk/VS-PETS/TESTING/CAMERA3

${ }^{2}$ http://www.cvg.rdg.ac.uk/PETS2006/data.html
}

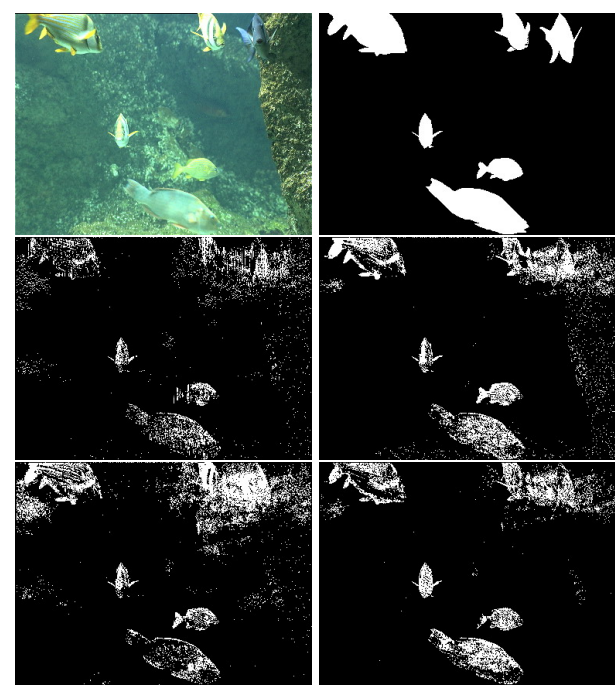

Figure 1. First row: Current image, Ground truth. Second row: Sugeno-Ohta, ChoquetOhta. Third row: Choquet-HSV, ChoquetYCrCb.

\begin{tabular}{|l||l|l|l|l|}
\hline $\begin{array}{l}\text { Integral } \\
\text { Color Space }\end{array}$ & $\begin{array}{l}\text { Sugeno } \\
\text { Ohta }\end{array}$ & $\begin{array}{l}\text { Choquet } \\
\text { Ohta }\end{array}$ & $\begin{array}{l}\text { Choquet } \\
\text { HSV }\end{array}$ & $\begin{array}{l}\text { Choquet } \\
\text { YCrCb }\end{array}$ \\
\hline \hline Similarity rate & 0.27 & 0.44 & 0.34 & 0.46 \\
\hline
\end{tabular}

Table 1. Similarity measure

\subsection{Aqu@theque dataset}

This dataset contains several video sequences presenting fishes in tank. The goal of the application Aqu@ theque [4] is to detect fishes and identify them. In these aquatic video sequences, there are many critical situations. For example, there are illumination changes owed to the ambient light, the spotlights which light the tank from the inside and from the outside, the movement of the water due to fish and the continuous renewal of the water. Furthermore, the constitution of the aquarium (rocks, algae) and the texture of fishes amplify the consequences of the brilliant variation. Figure 1 shows the experiments made on one sequence. It is noticed that the results obtained using the proposed method are better than using the method proposed by [17] with the same color space, i.e Ohta. The results obtained with the Choquet integral using other color spaces, i.e the HSV and $\mathrm{YCrCb}$ confirme that optimum results are obtained using Choquet integral with texture and the $\mathrm{YCrCb}$ color features. The evaluation has been done quantitatively using a similarity measure, derived by $\mathrm{Li}$ et al. [8], between detected region and the corresponding ground truth. Table 1 shows 
the similarity value obtained for the previous experiments. It is well identified that optimum results are obtained by the proposed method.

\subsection{VS-PETS dataset}

The dataset is formed by outdoor scenes (soccer video sequences). The first column of Figure 2 shows the results obtained with the method proposed by [17] and with the Choquet integral using the $\mathrm{YCrCb}$ color space. The silhouettes are better detected and the illumination variations on the white border are less detected using our method.

\subsection{PETS 2006 dataset}

The algorithm has been tested also on PETS 2006 benchmark data for an indoor sequence in video surveillance context. Once again the use of Choquet integral with $\mathrm{YCrCb}$ color space shows a robustness to illumination changes and shadows, as we can see in the second column of Figure 2.
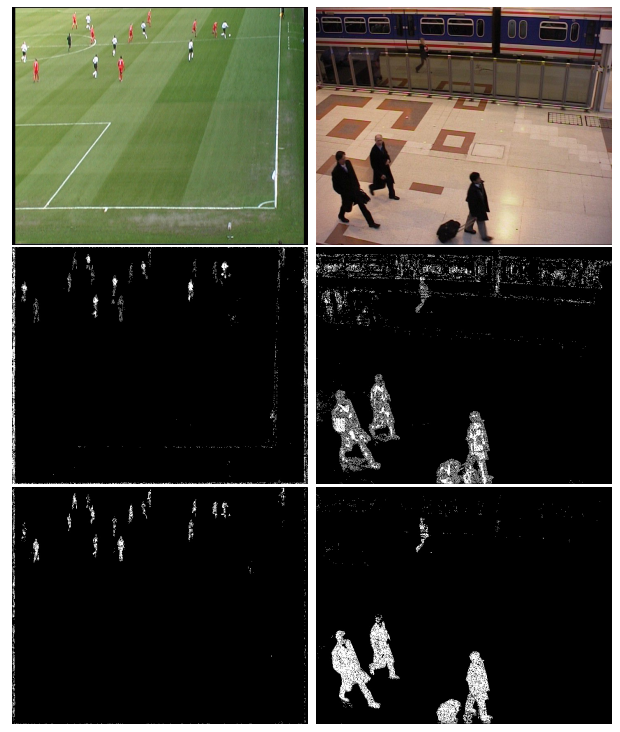

Figure 2. First row: Current image. Second row: Sugeno-Ohta. Third row: Choquet$\mathrm{YCrCb}$.

\section{Conclusion}

In this paper, we have presented a foreground detection method using the Choquet integral for fusing color and textures features. Experiments in multimedia and video surveillance datasets show that Choquet integral with $\mathrm{YCrCb}$ color space is robust against some changes in background. Further research consist in fusing other features.

\section{References}

[1] J. Carranza, M. Magnor. Free-viewpoint video of human actors. ACM Transactions on Graphics, 22(3):569-577, 2003.

[2] R. Chang, T. Ghandi. Vision modules for a multi sensory bridge monitoring approach. ITSC 2004, 2004.

[3] S. Cheung, C. Kamath. Robust background subtraction with foreground validation for urban traffic video. Journal of Applied Signal Processing, EURASIP, 2005.

[4] F. El Baf, T. Bouwmans. Comparison of background subtraction methods for a multimedia learning space. International Conference on Signal Processing and Multimedia, SIGMAP, July 2007.

[5] A. Elgammal, L. Davis. Non-parametric model for background subtraction. 6th European Conference on Computer Vision, ECCV, June 2000.

[6] M. Heikkila, M. Pietikinen. A texture-based method for modeling the background and detecting moving objects. IEEE Transactions on Pattern Analysis and Machine Intelligence, PAMI, 28(4):657-662, 2006.

[7] B. Lee, M. Hedley. Background estimation for video surveillance. Image and Vision Computing New Zealand, IVCNZ, pages 315-320, 2002.

[8] L. Li, W. Huang. Statistical modeling of complex background for foreground object detection. IEEE transaction image processing, 13(11):1459-1472, November 2004.

[9] N. McFarlane, C. Schofield. Segmentation and tracking of piglets in images. British Machine Vision and Applications, pages 187-193, 1995.

[10] S. Messelodi, C. Modena. A kalman filter based background updating algorithm robust to sharp illumination changes. ICIAP 2005, September 2005.

[11] Y. Narukawa, T. Murofushi. Decision modelling using the choquet integral. Modeling Decisions for Artificial Intelligence, 3131:183-193, 2004.

[12] C. Stauffer. Adaptive background mixture models for realtime tracking. Proceedings IEEE Conference on Computer Vision and Pattern Recognition, CVPR, pages 246252, 1999.

[13] M. Sugeno, S. Kwon. A new approach to time series modeling with fuzzy measures and the choquet integral. 4th IEEE International Conference on Fuzzy Systems, pages 799-804, March 1995.

[14] H. Tahani, J. Keller. Information fusion in computer vision using the fuzzy integral. Transaction on Systems, Man, and Cybernetics, 20(3):733-741, 1990.

[15] K. Toyama, J. Krumm. Wallflower: Principles and practice of background maintenance. International Conference on Computer Vision, September 1999.

[16] C. Wren, A. Azarbayejani. Pfinder : Real-time tracking of the human body. IEEE Transactions on Pattern Analysis and Machine Intelligence, 19(7):780 -785, July 1997.

[17] H. Zhang, D. Xu. Fusing color and texture features for background model. Third International Conference on Fuzzy Systems and Knowledge Discovery, FSKD, 4223(7):887893, September 2006.

[18] J. Zheng, Y. Wang. Extracting roadway background image: A mode based approach. TRB 2006, 2006. 